\title{
Transformaciones en el comportamiento en función del género en la vejez: el caso de las relaciones familiares actuales entre abuelas, abuelos y nietas y nietos
}

\section{Rita Radl Philipp}

Universidade de Santiago de Compostela. Facultade de Ciencias de la Educación Campus Sur. Santiago de Compostela

cpritausc.es

\section{Resumen}

Esta exposición suscita la problemática de las transformaciones de las relaciones de género en la vejez a través de una investigación reciente de las relaciones familiares actuales entre abuelas, abuelos, nietas y nietos. Se aportan unos primeros datos que muestran sobre todo un cambio en las tareas y funciones tradicionales del cuidado que son asumidas cada vez más por los varones en la vejez.

Palabras clave: roles de género, vejez, familia, procesos de cambio social, abuelas y abuelos.

Abstract. Behaviour transformations as regards gender in the old age: the case of present family relations between grandmothers, grandfathers, grandchildren (boys and girls)

This exposition tries the theme of the transformation on gender relationship in aging. It starts from a recently research about family relationships between grandmothers, grandfathers and young boys and girls. The research data seems changing process in the traditional tasks and functions of nursing assumed by grandfathers.

Key words: Gender roles, aging, family, process of social change, grandmothers and grandfathers.

\section{Sumario}

Introducción 4. Evaluación de los datos: un avance

1. Cambio social, vejez y relaciones $\quad 5$. A modo de conclusión familiares Bibliografía

2. Aspectos teóricos e hipótesis central

3. Planteamiento metodológico

\section{Introducción}

Un tema ya recurrente, tanto en foros científicos como en los de índole social, político y económico, es el de la vejez en la actualidad, el alto índice del envejecimiento de la estructura poblacional y sus repercusiones en las sociedades industriales modernas. Esta problemática preocupa por el nivel creciente del número de personas mayores en estas sociedades, que, al mismo tiempo unido 
a unas cifras estrepitosamente bajas de los nacimientos, modifica por completo su estructura poblacional. Y efectivamente parece que se trata de un problema que empieza a suscitar en los muy diversos campos del conocimiento un interés especial, muy particularmente en todas aquellas parcelas vinculadas a la sociología.

Un aspecto hasta ahora todavía apenas indagado es el referido a las relaciones efectivas entre los hombres y las mujeres ancianos o, dicho en otras palabras, cómo se reparten las tareas, funciones y trabajos, qué rasgos específicos muestran los comportamientos en función del género y en qué medida afectan éstos a las definiciones de los roles de género femenino y masculino de las personas mayores. Esta cuestión no olvida ni minimiza en absoluto el hecho y las repercusiones sociales de la feminización de la vejez ${ }^{1}$, pero en realidad se centra en cuestiones que van mucho más allá de esta primera.

Desde múltiples puntos de vista, la familia es, de forma evidente, la institución más afectada por la situación actual de la vejez y, consecuentemente desde una perspectiva de género en un sentido amplio, las relaciones familiares en la vejez tienen especial relevancia. Muy concretamente y en consonancia con los datos que se han obtenido en el contexto internacional en estudios recientes sobre las abuelas y sus nuevas relaciones familiares, éstas asumen cada vez más los valores de la autorrealización, independencia y autonomía personal en el trato con las nietas, los nietos y la familia.

De modo explícito, nos situamos aquí en el contexto señalado con el propósito de abordar el complejo temático de las nuevas relaciones familiares entre abuelas, abuelos, nietas y nietos como un caso particular de las transformaciones de los roles de género en la vejez. Profundizaremos en las características de las relaciones familiares entre abuelas, abuelos, nietas y nietos como un caso específico de las transformaciones de los roles de género en la vejez, con vistas a la elaboración de categorías clasificatorias al respecto. Esto es, perseguimos detectar si los abuelos y las abuelas desempeñan sus roles de género tradicionales - las abuelas, su papel afectivo y de cuidado, mientras que los abuelos ejercen su papel instrumental vinculado a su experiencia laboral—o bien si los abuelos y las abuelas actuales incorporan elementos de género nuevos en su contacto con los nietos y las nietas y en su vida familiar de acuerdo con la tesis de que la vejez sirve de hecho para compensar las carencias vividas en las experiencias humanas y sociales por las personas mayores en etapas anteriores de su vida. Por razones intracientíficas, nos referiremos a nuestro contexto sociocultural presentando unos primeros datos empíricos sobre la cuestión que

1. Es bien sabido como este fenómeno incluso lleva a propuestas aberrantes en cuanto a cómo solucionar la problemática de las prestaciones sociales (pensiones) en la vejez como muestra la actual discusión acerca de un proyecto de ley en Suecia que pretende recortar las cantidades correspondientes de las pensiones a las mujeres, puesto que tienen una mayor esperanza de vida. Recientemente, algún diputado español ha intentado introducir la misma idea en el contexto político del país, pero afortunadamente ha fracasado, al menos por el momento. 
corresponden a una investigación pionera que se está llevando a cabo acerca del tema en el contexto español ${ }^{2}$.

Ahora bien, como cabe señalar muy especialmente, la relevancia del proyecto que constituye el núcleo de nuestras reflexiones en este artículo se deriva del hecho de ser uno de los primeros estudios sobre las transformaciones de los roles de género en la vejez que incluye además expresamente una perspectiva referida a los varones y, por tanto, datos sobre el colectivo masculino. Analizamos la relación de los abuelos con los nietos y las nietas indagando en los cambios del rol masculino en función del género en la vejez, aparte de analizar el comportamiento femenino a través de la figura de la abuela. En este sentido, el significado de la investigación desde un ángulo sociológico se desprende ante todo de dos aspectos. En primer lugar, del valor de las transformaciones en las relaciones de género en la vejez, que traspasan el ámbito propio de las relaciones privadas y familiares por múltiples motivos; adquieren desde un punto de vista sociológico-global el valor de unos cambios estructurales. Y, en segundo lugar, del hecho de que la vejez prolongada se convierte en una posibilidad real de compensación de carencias en la experiencia en función del género de hombres y mujeres mediante el desempeño de su rol de abuelas y abuelos en un plano hipotético simétrico de funciones compartidas. Tanto los cambios estructurales con respecto a la vejez como los nuevos parámetros demográficos constituyen aquí el trasfondo social más relevante.

\section{Cambio social, vejez y relaciones familiares}

El tema del envejecimiento de la población de las sociedades industriales modernas constituye un tema cada vez más importante por las repercusiones que parece tener en la organización y estructura social, sobre todo en cuanto a las alteraciones del estado de bienestar que conllevan los cambios que se están produciendo actualmente. El alto índice de envejecimiento de la población constituye, de hecho, una problemática que afecta a la estructura misma de las sociedades, a todas sus instituciones y organizaciones. Conlleva un cambio en la estructura poblacional, en la pirámide de la población, de los países industriales actuales que deja prever múltiples consecuencias para el estado de bienestar social que afecta de modo especial al así llamado "contrato generacional» y a las relaciones familiares. La familia, según nuestra tesis, es la institución más afectada por los cambios producidos por el así llamado «envejecimiento de la población» por múltiples motivos y en diversos aspectos; es la instancia que está en un contacto permanente con la vejez a través de sus miembros.

2. Esta investigación dirigida por Rita Radl Philipp está financiada por la Xunta de Galicia dentro del plan nacional I+D bajo el título: "Transformaciones de las relaciones familiares en la vejez en función del género. El caso particular de las relaciones entre abuelas, abuelos, nietas y nietos» (PGIDT99PX121401B). El equipo de investigación está integrado por Constanza Tobío Soler, Jorge García Marín, Ma Begoña Vázquez Gómez, Carolina García Borrazás, Ma García Añon y Marta Poncet Souto. 
Según los datos, un 61\% de las personas mayores de 65 años en España mantiene un contacto diario con su familia (Walker, 1996), es decir, con hijos, hijas, nietas, nietos, hermanos, etc. Este índice corresponde en el contexto europeo a la mayor frecuencia de los contactos con la familia, sólo superado por los ancianos en Italia. De este hecho se deriva la especial importancia de investigar las nuevas relaciones familiares en la vejez en nuestro contexto sociocultural, que, en el caso que nos ocupa, se refiere a los cambios en el comportamiento en función del género de las abuelas y los abuelos actuales. Este complejo temático se sitúa de modo específico en el contexto de la socialización humana en un sentido muy amplio. Si entendemos, efectivamente, por socialización el proceso mediante el cual el "ser humano biológico» se convierte en ser social, este proceso incluye todas las experiencias del ser humano en cualesquiera de sus épocas vitales, esto es, desde la niñez hasta la tercera y cuarta edad. El descenso estrepitoso de los nacimientos y el aumento de la esperanza de vida de las personas mayores en las sociedades industriales modernas constituye un fenómeno comprobado que muestra ciertamente un envejecimiento y una modificación paulatinos de la estructura piramidal de estas sociedades. La tasa de mortalidad sobrepasa el índice de los nacimientos, y el así llamado «efecto tijera» se deja notar cada vez más. Tenemos ante nosotros los efectos de una renuncia más o menos consciente de la tradicional función del cuidado de la familia en relación con la generación de los jóvenes.

En concreto, España cuenta con el índice de fecundidad menos elevado en el conjunto de la Unión Europea, que se situaba en el año 2000 en el 1,22 frente al 1,20 del año 1999. En el conjunto europeo, los datos del año 2000 señalan que cada mujer en edad fértil da a luz a 1,53 niños (en el año 1999 el índice era del 1,45). Después de España, los índices más bajos corresponden a Italia $(1,25)$, Grecia $(1,30)$ y Alemania $(1,34)^{3}$, que son, en definitiva, los países con el más elevado nivel del envejecimiento poblacional, ya que están por debajo del nivel de reposición.

En el presente trabajo, defendemos además la tesis de que el envejecimiento de las sociedades industriales modernas, lejos de ser un fenómeno negativo, obliga de hecho a transformaciones y reorganizaciones fundamentales en los muy diversos ámbitos sociales, políticos, económicos y culturales, modificaciones que, por otra parte, pueden reconducir los efectos tan tremendamente instrumentalizadores y deshumanizadores de la dinámica social moderna. Esto significa una liberación consciente de las tareas del cuidado de la familia, ya que obedece en efecto a una decisión racional de la pareja. Sin embargo, en absoluto podemos pensar en una pérdida de la tradicional función del cuidado de la familia, ni siquiera con respecto a las generaciones jóvenes. La tradicional instancia de socialización primaria por excelencia no puede renunciar, ni renuncia, a ésta su función del cuidado de los jóvenes y niños. Eso sí, la asun-

3. Los datos han sido facilitados por la Oficina Europea de Estadísticas, EUROSTAT (2001), Bruselas. 
ción y distribución de estas tareas se están transformando y las modificaciones afectan no sólo a las relaciones de pareja, al rol del padre, de la madre, del hijo y de la hija en la estructura familiar, como por otra parte múltiples investigaciones han comprobado y a pesar de que los cambios en función del género no parecen ser tan esenciales como sería de desear, sino que también tienen consecuencias para los roles femenino y masculino en la vejez.

\section{Aspectos teóricos e hipótesis central}

La investigación que constituye el centro de nuestras reflexiones aquí se ocupa de dilucidar empíricamente la temática de las relaciones familiares en la vejez, de modo concreto las relaciones actuales entre abuelas, abuelos, nietas y nietos. Partimos de la base de que estas relaciones muestran, en efecto, aspectos realmente nuevos en comparación con los elementos tradicionalmente definitorios de las citadas relaciones entre abuelas, abuelos y nietas y nietos.

Nuestra hipótesis central parte del enunciado de que las abuelas y los abuelos actuales siguen cumpliendo una función básica en cuanto al cuidado de nietos y nietas se refiere. Con esto prestan un apoyo importante a la familia, no sólo en el caso de hacer posible en gran medida la introducción de las jóvenes madres en el mercado laboral, tal y como ha sido comprobado en diferentes estudios sobre el tema (Sommer-Himmel, 2000; Attias-Donfut, 2001; Tobio, 2001; Gessat-Anstett, 2001) ${ }^{4}$, sino que se hacen cargo de tareas que son propias de las funciones del cuidado. Sin embargo, observamos como la configuración concreta de las relaciones entre abuelas, abuelos, nietas y nietos muestran indicios de estar inmersas en un profundo proceso de cambio, sobre todo en lo que respecta a los roles de género tradicionales. Aquí defendemos la tesis de que las abuelas y los abuelos actuales incorporan elementos de género nuevos en su contacto con los nietos y las nietas y en su vida familiar. Desempeñan sus roles de género tradicionales, esto es, las abuelas su papel afectivo y de cuidado, mientras que los abuelos ejercen su papel instrumental vinculado a su experiencia laboral. Al lado de estos cometidos se percibe además como a la vez los abuelos asumen tareas del cuidado en relación con sus nietos - tareas que no habían ejercido realmente antes en relación con sus hijos-y las abuelas rechazan en parte las funciones clásicas del cuidado insistiendo en la importancia de un espacio propio y de autonomía personal. Precisamente estos aspectos han sido señalados como elementos decisivos que apuntan a un proceso de cambio en el tradicional rol del cuidado de las abuelas en otros contextos socioculturales. Tal idea está en consonancia con los datos aportados por los estudios

4. En concreto, citamos los trabajos de Constanza Tobio: TobIO, C. (2001), «En Espagne, la abuela au secours des mères actives», en ATTIAS-Donfut, C. y SEgALEN, M. (eds.), Le siécle des grand-parents, Éditions Autrement, París, p. 102-115, y ToBIO, C. (2001), «La familia y el empleo: prácticas y discursos de las mujeres», en RADL PHILIPP, R. (ed.), Cuestiones actuales de sociología del género. CIS-Universidad de Santiago de Compostela, Madrid, p. 129-148. 
científicos sobre la vejez procedentes de una temática muy variada. Los datos de una investigación piloto representativa de Herlyn y Kistner de 1998, y de Kivett de 1991, sobre las transformaciones con respecto al rol de las abuelas en el seno familiar del ámbito germano constatan una «socialización doble» de las abuelas más jóvenes que apunta a contenidos vitales nuevos en su vejez. Este hecho se manifiesta en un cierto distanciamiento y menor contacto con sus nietos y que estas abuelas muestran a la vez una orientación hacia la familia y la profesión. Como otra clase de abuelas aparecen las de más edad, que están primordialmente centradas en la familia y en la función tradicional de la abuela, asumiendo las tareas y los quehaceres del cuidado con los nietos.

De modo específico, pretendemos dilucidar las modificaciones que atañen a los comportamientos de género de las abuelas, que, según los trabajos citados en el contexto internacional (Herlyn y Kistner, 1998; Kivett, 1991), asumen cada vez más los valores de autorrealización, independencia y autonomía personal en el trato con los nietos, las nietas y la familia. Por otra parte, pretendemos determinar si el rol desempeñado por los abuelos corresponde asimismo a la asunción de tareas y funciones nuevas, en su caso de trabajos de cuidado con los nietos y nietas en el seno familiar, tema que hasta el momento no ha sido suscitado de modo explícito en el contexto de los estudios mencionados.

Así, podemos referir aquí, siguiendo a Kirstner y Kevitt, que al menos es posible diferenciar al lado de la abuela «tradicional», dos tipos distintos más. En concreto, el tipo de abuela que busca su autonomía personal renunciando a su rol tradicional. Este modelo anhela cada vez más su autorrealización y defiende su independencia y autonomía personal en el trato con los nietos, las nietas y la familia. Aparte de este prototipo, existe la abuela que asume ciertos aspectos del cuidado en relación con los nietos, pero a la vez introduce elementos nuevos de un rol apoyado en una concepción de autonomía personal.

En suma, los datos muestran la existencia paralela de tres modelos de rol de la abuela. En primer lugar, el modelo tradicional de la abuela centrado en la familia (el cuidado de la familia, de los hijos y de los nietos es prioritario). En segundo lugar, el modelo de la abuela que compagina al mismo tiempo valores e intereses intrafamilares y extrafamiliares, y, por último, el modelo de la abuela centrada en valores extrafamiliares (con la prioridad de la independencia, autonomía, etc.). De modo global, sobresale en los resultados un trato especialmente positivo entre abuelas y nietos en los casos en los cuales las abuelas muestran preferentemente unos contenidos de roles de género nuevos (autonomía, autorrealización fuera de la familia, etc.), y éste es un aspecto que se constata cada vez con más frecuencia. Es más, las autoras argumentan que precisamente estos contenidos de roles de género nuevos repercuten muy positivamente en las relaciones intergeneracionales, contribuyendo además a una gran satisfacción personal de las abuelas que efectivamente compensan, tal y como se describe detalladamente en la parte cualitativa de las investigaciones, sus experiencias vitales anteriores en la familia. En cuanto a otras variables habituales en este tipo de estudios, se mantienen las correlaciones positivas de 
los datos en relación con las variables clase, actividad laboral, estado de salud y distancia kilométrica entre el lugar de residencia de los abuelos y los nietos constatadas en otros estudios procedentes del ámbito anglosajón (Pfeifer y Sussman, 1994).

En lo que se refiere al núcleo temático de nuestra investigación, defendemos la tesis que en nuestro contexto sociocultural, con un comportamiento diferencial en función del género muy acusado en la generación de las personas mayores, el rol desempeñado por los abuelos corresponde a funciones instrumentales y técnicas, por ejemplo, reparar juguetes, enseñar a construir algún objeto, manejar la bicicleta, etc., pero también a tareas del cuidado tales como llevar las nietas y los nietos de paseo, recogerlos en el colegio, darles la merienda, etc. Perseguimos comprobar si el rol desempeñado por los abuelos incluye efectivamente la asunción de las tareas y funciones del cuidado con los nietos y las nietas en el seno familiar. Esperamos de nuestros datos que nos permitan confirmar nuestras hipótesis. $\mathrm{O}$, dicho en otras palabras, nuestro interés especial consiste en poder confirmar los modelos de abuelas citadas y poder confirmar la existencia de un nuevo rol de los abuelos en cuanto a la incorporación de elementos clásicos del cuidado en su relación con los nietos, hecho que apuntaría, según nuestra opinión, a la existencia de un proceso de transformación y modificación en el modelo de los roles de género en la vejez. Este último tema apenas ha sido investigado hasta el momento, ni en nuestro contexto sociocultural, ni en los países de nuestro entorno europeo, según es sabi$\mathrm{do}^{5}$. Asimismo, pretendemos elaborar una tipología referente al rol desempeñado por los abuelos que contemplase los modelos concretos con los aspectos transformativos más relevantes de cara a los roles de género masculino en la vejez, concretado en el rol generacional y de género del abuelo.

En definitiva, consideramos que la vejez, sin duda prolongada, debido a los logros y las configuraciones específicas que ha adquirido en las sociedades modernas del bienestar, permite a los sujetos una «compensación» de sus experiencias vitales ${ }^{6}$, hecho que atañe asimismo al comportamiento en función del género de las personas mayores, muy particularmente en relación con su rol de abuelo o abuela en el seno familiar. Con esto se convierte la vejez prolongada en un espacio para el desempeño de los roles generacionales y de género de abuelas y abuelos en un plano hipotético-simétrico de funciones compartidas. Esta lectura parte de una consideración eminentemente positiva de la vejez.

5. Una excepción constituye aquí el trabajo de Claudine Attias-Donfut: «La fabrication des grands-péres», en AtTiAs-Donfut, C. y SEGALEN, M. (eds.), Le siécle des grand-parents, Éditions Autrement, París, p. 48-73.

6. Véase, por ejemplo, como las personas mayores son cada vez más una población potencial para el sector turístico en los países industriales y esto no solo porque disponen de tiempo libre, sino también debido a que no han tenido apenas esta oportunidad en otras épocas de su vida. El caso de la edad media de los turistas que visitan España procedentes de Alemania, de los países europeos nórdicos y de Estados Unidos corroboran esta tesis. 


\section{Planteamiento metodológico}

Por motivos de complementación informativa, se ha decidido realizar la recogida de la información con respecto a nuestro tema a partir de dos muestras, a saber, de una muestra de personas mayores (abuelas y abuelos) y de una muestra de jóvenes (nietas y nietos). En estos momentos tenemos acceso a los datos de la primera muestra que ofrece los datos correspondientes de las abuelas y de los abuelos. El instrumento utilizado para la recogida de los datos ha sido un cuestionario cuya elaboración se efectuó después de la realización de varias sesiones de grupos de discusión sobre el tema de la situación actual de las relaciones entre abuelas, abuelos, nietas y nietos con personas mayores (en concreto con alumnos y alumnas del así llamado cuarto ciclo universitario en la Universidad de Santiago). Después de la validación del cuestionario construido en una muestra piloto, se ha elaborado la versión definitiva.

Teniendo en cuenta la dificultad en el manejo de cuestionarios para las personas mayores, los cuestionarios han sido presentados mediante contacto personal con un entrevistador a una muestra aleatoria de $111 \mathrm{~N}$. La distribución por género muestra una mayoría de mujeres, 79 (71,2\%), frente a 32 hombres $(28,8 \%)$, tal y como se aprecia en el gráfico 1 . Esta mayor proporción femenina se explica por la propia composición de los grupos de la tercera y cuarta $\mathrm{edad}^{7}$, en que las mujeres superan siempre a los hombres. Es impor-

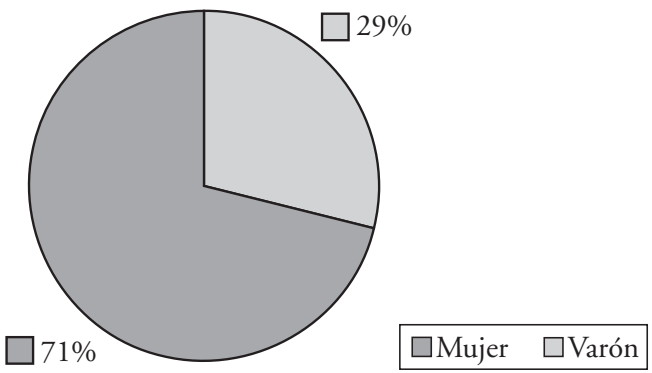

Gráfico 1. Muestra según sexo.

Fuente: elaboración propia.

7. Se trata, efectivamente, de un hecho comprobado que, a partir de los 60 años, el número de mujeres siempre supera con creces al de varones. En 1991, aproximadamente un 60\% de los ancianos eran mujeres. Este fenómeno se debe especialmente a la sobremortalidad en los varones, que es superior a la de las mujeres en todas las edades, incluso ya en la franja de edad de 15 a 25 años, donde se sitúa en el índice 3. En la edad adulta, esto es, de 25 a 65 años, este índice es del 2,5; de 65 a 74 años es del 2 e incluso persiste aún con un promedio de un $10 \%$ de mayor mortalidad para los varones entre los 75 y los 90 años. El índice de sobremortalidad únicamente se iguala para varones y mujeres a los 90 años de edad (Santos del Campo, 1996, p. 188). 
tante señalar que nuestras tablas de contingencia nos indican además un 87,8\% de viudas, frente a un $12,2 \%$ de viudos.

La información recogida ha sido codificada en el programa SPSS, en tablas de contingencia. El índice de error ha sido mínimo, según nuestros cálculos.

En este espacio sólo haremos especial hincapié en aquellos datos que nos parecen más sobresalientes y que efectivamente se entienden en relación con nuestras hipótesis. Como principio de comprobación asumimos especialmente el principio de la comprobación negativa, esto es, el de la falsación.

\section{Evaluación de los datos: un avance}

Comenzamos por comentar que la mayoría de las abuelas, el 55,7\%, señalan como su profesión la de ama de casa, mientras que un 31,3\% de los abuelos eran empleados de empresas y un 18,8\% ejercían su actividad laboral en la agricultura y la pesca. Sigue con un $12,5 \%$ el grupo de los trabajadores autónomos (gráfico 2). La mayoría de los entrevistados y entrevistadas pertenecen al grupo sin estudios y los estudios primarios (gráfico 3).

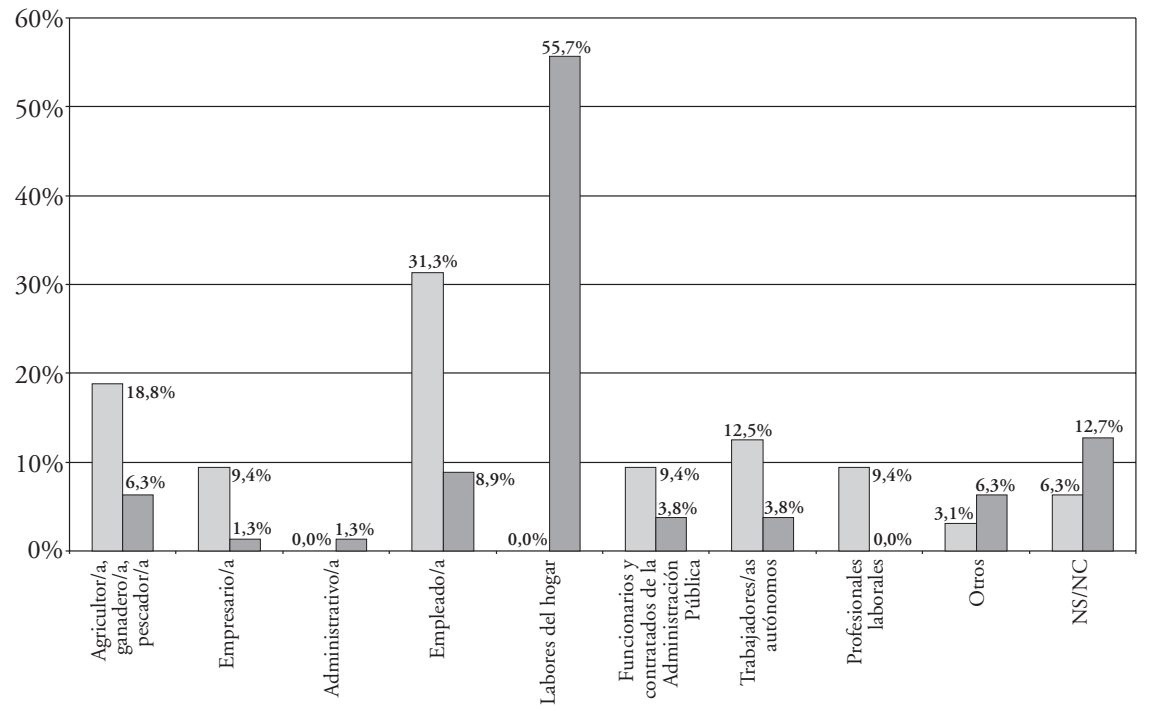

$\square$ Mujer $\square$ Varón

Gráfico 2. Profesión anteriormente ejercida según sexo.

Fuente: elaboración propia. 


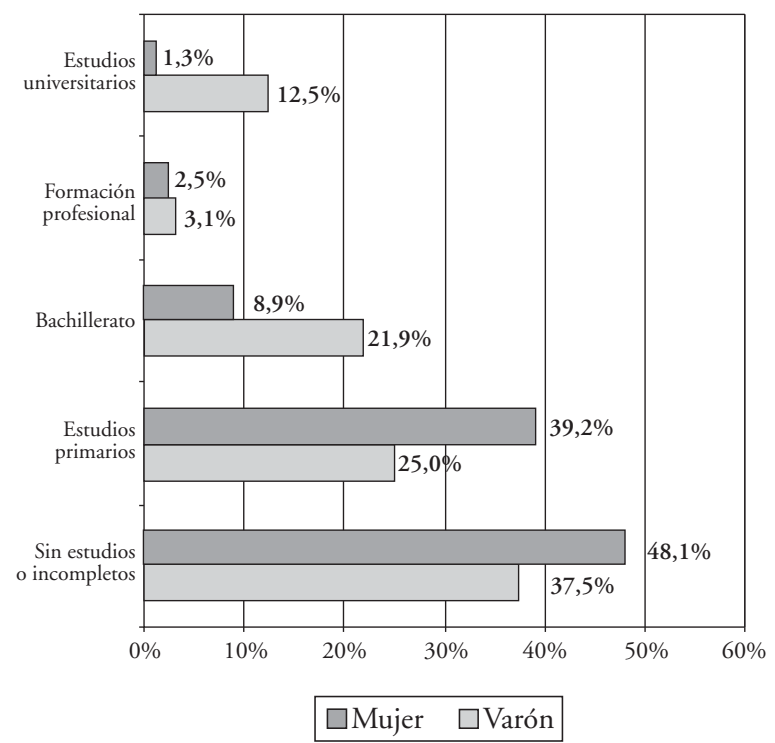

Gráfico 3. Estudios realizados.

Fuente: elaboración propia.

Una de las cuestiones más relevantes, si no la que más, es la referida a las actividades efectivas que realizan (o realizaban) los abuelos y las abuelas con los nietos y las nietas. En el gráfico 4 vemos los datos referentes a las nietas y el gráfico 5 recoge los valores válidos para los nietos. Los abuelos indican casi en un $72 \%$ de los casos que lo que más hacen con nietas y nietos es jugar y darles dinero (en un $65,6 \%$ y un $68,8 \%$ respectivamente), contarles cuentos (en un $50 \%$ y un $46,9 \%$ ) y llevarles al parque o en coche. Tanto la penúltima actividad como contar cuentos es algo que se hace en mayor medida con las nietas que con los nietos. No sabemos si es porque simplemente hay más nietas que nietos o es debido a algún otro factor. Podríamos decir que los abuelos se encargan más de actividades lúdicas. Destaca especialmente que las abuelas mantienen sobre todo las tareas clásicas del cuidado en el seno familiar, esto es, se encargan de darles la comida a las nietas (en un $70,9 \%$ de los casos) y nietos (en un $55,7 \%$ de los casos), les dan la merienda, la cena y el desayuno y los visten (gráficos 4 y 5). Asimismo, son tareas de la abuela bañar a los nietos y nietas y cambiar los pañales. Éstas últimas son funciones que los abuelos apenas asumen. Como diría un abuelo en un grupo de discusión, «el abuelo y la abuela se encargan conjuntamente de cuidar a los nietos y nietas, pero las abuelas se encargan sobre todo de las tareas alrededor de la comida y el abuelo de entretenerlos». En esta línea de argumentación es interesante mencionar como se mantiene la función de construir o reparar 


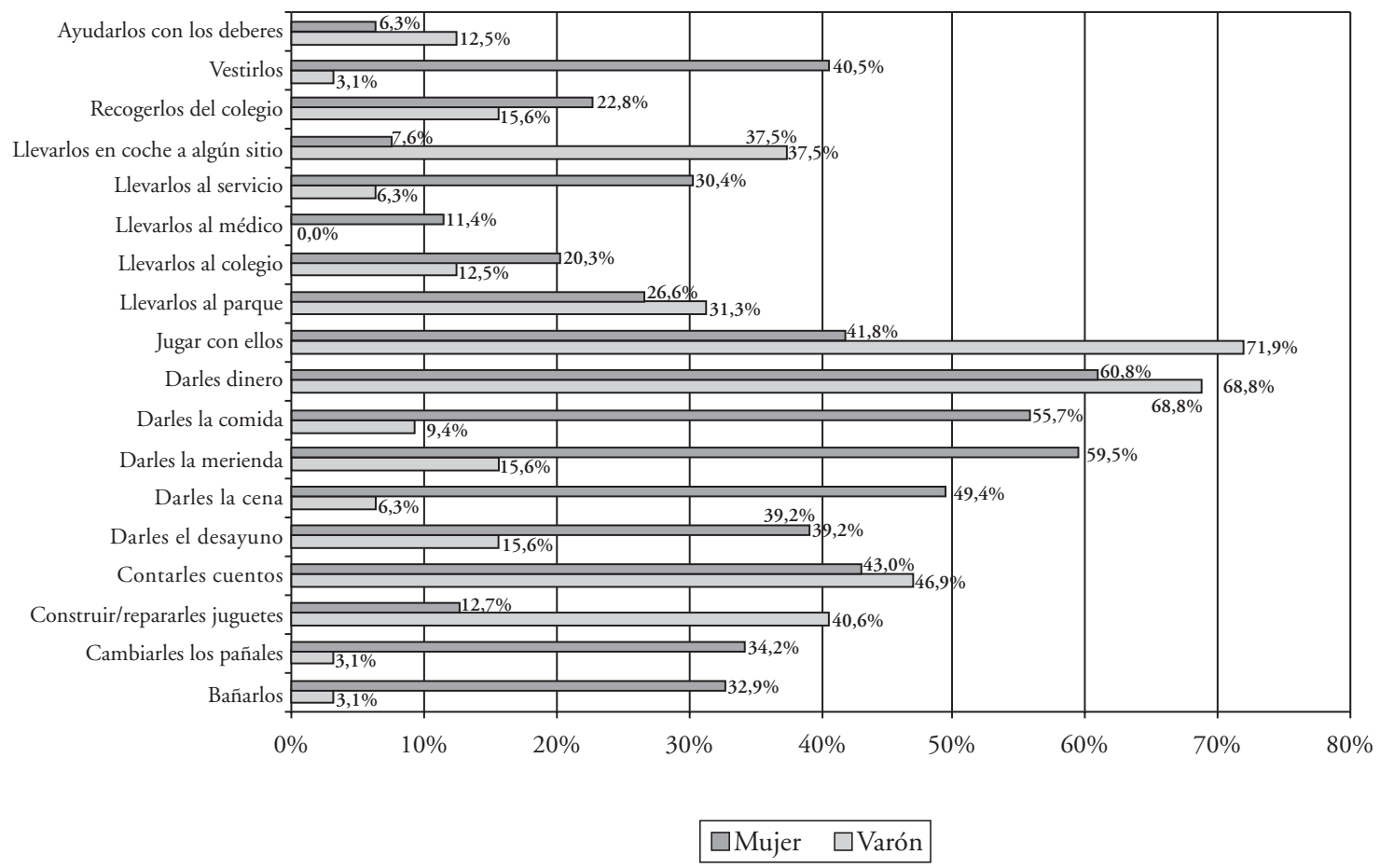

Gráfico 4. Actividades que realiza con sus nietos según sexo.

Fuente: elaboración propia. 


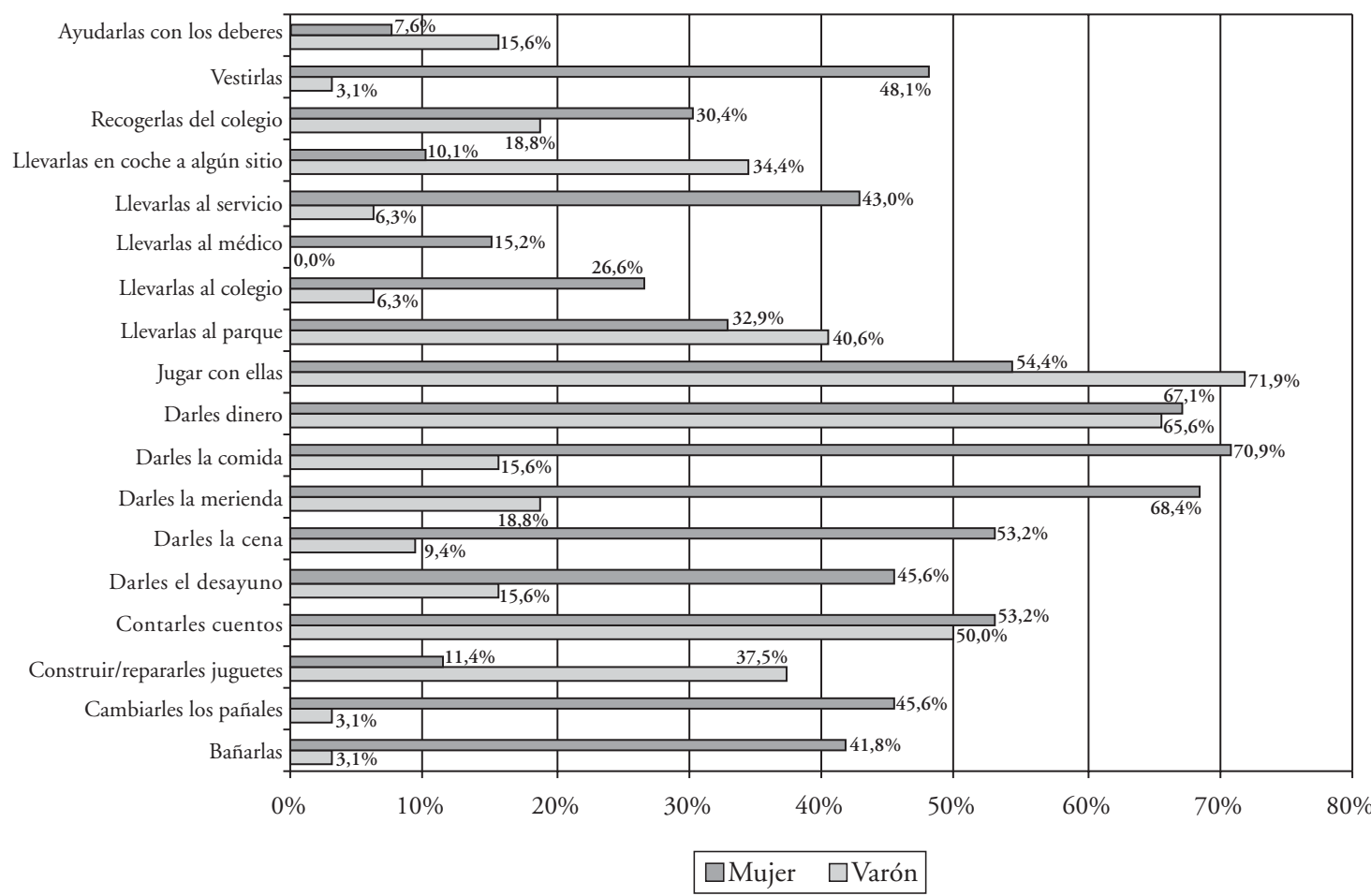

Gráfico 5 . Actividades que realiza con sus nietas según sexo.

Fuente: elaboración propia. 


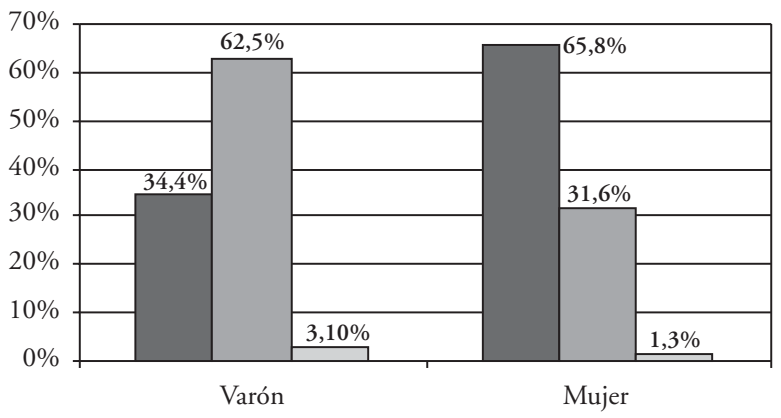

Sí $\square$ No $\square$ NS/NC

Gráfico 6. ¿Realizaba las mismas actividades con sus hijas e hijos?

Fuente: elaboración propia.

juguetes como una tarea instrumental y técnica de los abuelos (en un 37,5\% y un $40,6 \%$ respectivamente).

Viendo este apartado de las actividades en conjunto, hemos de señalar que predominan, en definitiva, para las abuelas las tareas clásicas del cuidado alrededor del cuidado personal (vestir, bañar, etc.) y de la comida. Estos quehaceres los empiezan a asumir también los abuelos, pero de forma más bien tímida (con una media del 16\%). Donde se iguala prácticamente la actividad de abuelas y abuelos es en el tema económico y en la cuestión de contar cuentos, además de llevar nietas y nietos al parque. Suponemos además que es una actividad compartida de abuelas y abuelos.

El cuadro de los cambios que van incorporando abuelas y abuelos con respecto a sus roles de género se complementa observando los datos en relación con el ítem: «¿Realizaba las mismas actividades con sus hijas e hijos?» (gráfico 6). Sólo un 34,4\% de los varones contesta que sí frente a un 65,8\% de las abuelas. Vemos como aquí el rol tradicional de la madre (abuela) prevalece, mientras que en el caso de los abuelos sí percibimos un cambio en las funciones del cuidado, por muy poco acentuadas que tengamos que considerar estas transformaciones.

La persistencia del rol tradicional de las abuelas se deduce también de los datos del gráfico 7 . Aquí casi el $60 \%$ de los varones y el $57 \%$ de las mujeres siguen manteniendo que la abuela sabe mejor que el abuelo lo que necesitan las nietas y los nietos. Sin embargo, más del $28 \%$ de los abuelos y de las abuelas aducen que esto no es así, hecho que muestra ciertamente una ambivalencia y desde luego unas definiciones de roles de género que se caracterizan por indicios de flexibilidad y grados de apertura.

En cuanto al ocio de abuelas y abuelos, lo que tiene una preferencia clara es pasear, tanto para la abuela como para el abuelo, y el estar con los nietos no ocupa los primeros lugares de las actividades lúdicas, se considera como un 


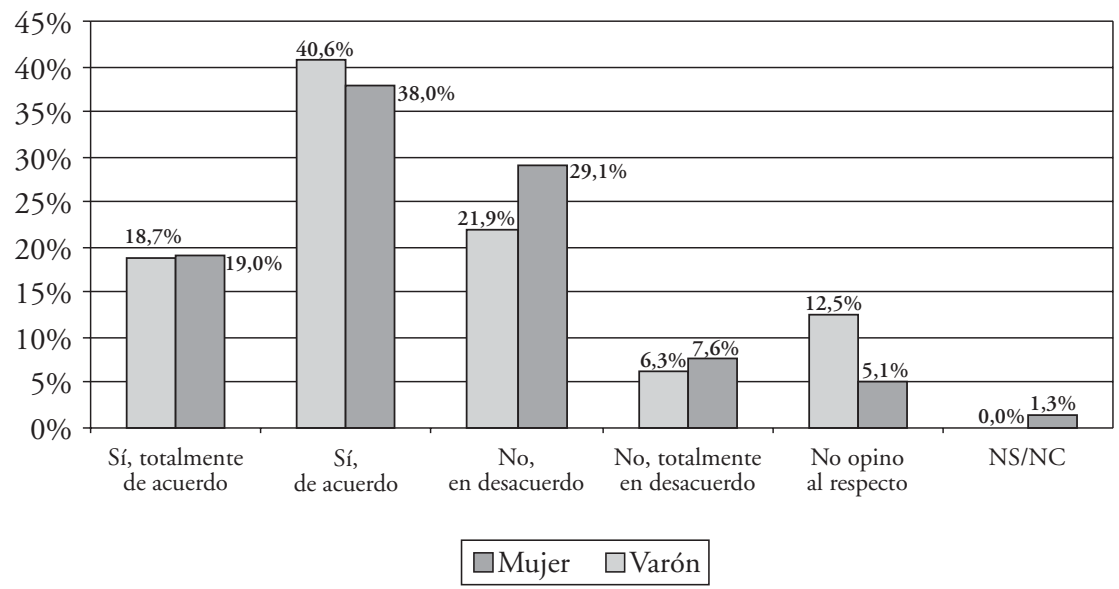

Gráfico 7. La abuela sabe mejor qué necesitan las nietas y los nietos que el abuelo. Fuente: elaboración propia.

deber y, a veces, como una obligación familiar. Quizás tenemos aquí ante nosotros aspectos que apuntan precisamente a unas actividades cuyo fin consiste, ante todo, en un ejercicio de autonomía personal. Las abuelas indican en un 50,6\% el viajar como una actividad preferente, en mayor medida que los abuelos $(28,1 \%)$, mientras que el estar con los nietos lo declaran sólo un $22,8 \%$ de las abuelas frente del 28,1\% de los abuelos. Estos datos inciden en nuestra afirmación anterior de que las abuelas pretenden salir del ámbito doméstico buscando un ejercicio de autonomía. En cambio, parece que los abuelos compensan su experiencia familiar doméstica, que durante su vida profesional ha quedado corta. El estar con la familia que aparece en el 74,7\% de los casos de las abuelas (abuelos: $46,9 \%$ ) se puede entender como un dato contradictorio en este contexto, pero se puede interpretar también como querer estar con el abuelo que en la vida anterior poco estaba participando en la vida familiar. La actividad que ocupa el primer lugar en el tiempo de ocio es ver la televisión, una actividad clara de tipo personal y de entretenemiento.

Ahora bien, es evidente que la relación de las abuelas y los abuelos con las nietas y los nietos ha cambiado, esto lo corroboran los datos del gráfico 8, y también parece asumido que, efectivamente, el cuidado de nietas y nietos por parte de abuelas y abuelos es una ayuda económica importante para los hijos e hijas de éstos (gráfico 9) y probablemente lo es sobre todo para las jóvenes madres, dado que ayuda a compatibilizar el cuidado familiar con un trabajo extradoméstico, en la línea de lo que indican los datos científicos sobre la ayuda familiar de las abuelas, tanto a nivel estatal como europeo. 


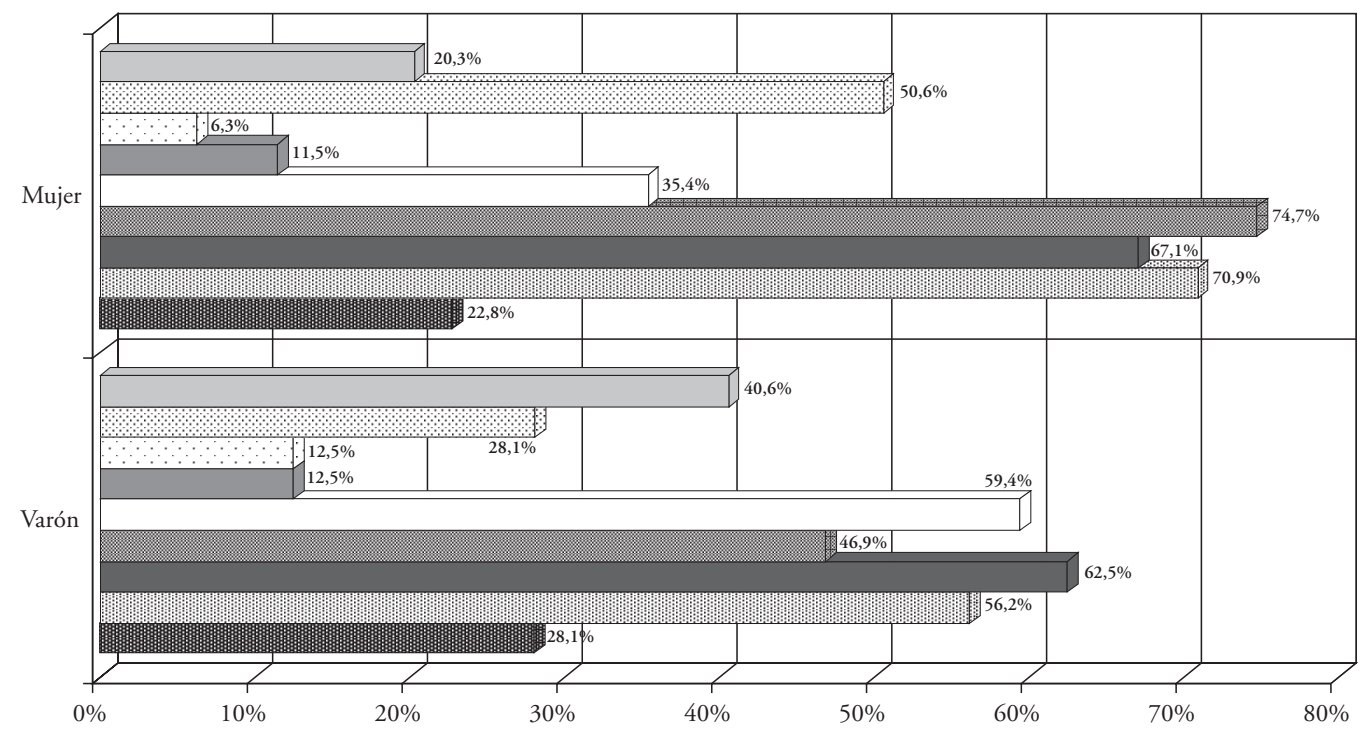

Gráfico 8. ¿Qué es lo que más le gusta hacer en su tiempo de ocio?

Fuente: elaboración propia.
17. Ver la televisión
Pasar el rato con la familia
$\square$ Ir al teatro, cine, concierto
Hacerme cargo de nietos y nietas

Asistir a una charla informativa

$\square$ Otros 


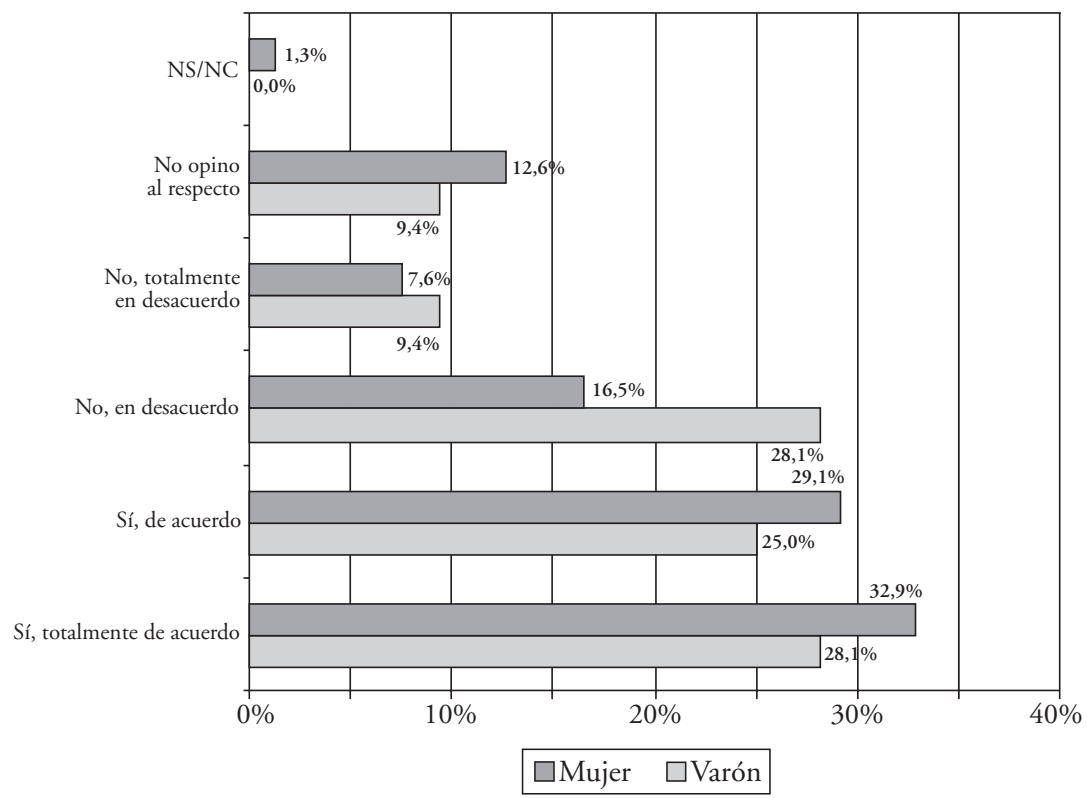

Gráfico 9. El cuidado de sus nietos y nietas es una ayuda económica importante para la familia de sus hijos e hijas.

Fuente: elaboración propia.

\section{A modo de conclusión}

Resumiendo, diríamos que es evidente que la relación de las abuelas y los abuelos con las nietas y los nietos ha cambiado, y que esta relación afecta a las concepciones de los roles de género en la vejez. Encontramos múltiples aspectos que resaltan que los varones asumen en su senectud tareas y funciones del cuidado y las valoran de modo distinto que en sus etapas de vida anteriores. Las mujeres siguen especialmente ejerciendo estas funciones, pero conjuntamente con los varones, sobre todo cuando aún vive la pareja. De este hecho deducimos que la experiencia en pos de una transformación de los comportamientos de género tradicionales es más positiva cuando los sujetos viven en pareja. También en los grupos de discusión las personas mayores insistían una y otra vez en la importancia de la colaboración entre abuelas y abuelos en cuanto a las tareas del cuidado con los nietos. Los hombres ponían de relieve que, aún cuando no tienen la misma práctica que sus mujeres en la cocina, poner la mesa, barrer, ir de recados, etc., sí eran funciones lógicas. Eso sí, parece que en estas tareas nietas y nietos más bien no colaboran. Contemplando el conjunto de estos primeros datos, parece, en efecto, que la vejez prolongada se convierte en un espacio para el desempeño de los roles generacionales y de género de abuelas y abuelos en un plano hipotético-simé- 
trico de funciones compartidas, aún cuando debemos advertir que el proceso de modificación de los roles de género más bien corresponde a un proceso lento y dificultoso, y esta aseveración es válida para la experiencia en función del género en la vejez. Evidentemente, estamos a la espera de los resultados que concierne a la muestra de nietas y nietos, esto es, esperamos que se complemente el panorama descrito a través del análisis de los datos obtenidos en esta muestra para ver si estamos en lo cierto con nuestras suposiciones en torno a la conducta efectiva de abuelas y abuelos. Una primera información, aún bastante superficial, apoya nuestras tesis.

\section{Bibliografía}

Attias-Donfut, C.; Segalen, M. (eds.). Le siécle des grand-parents. París: Éditions Autrement.

BAzO, M.T. (1990). La sociedad anciana. Madrid: CIS.

- (1994). «La familia como centro privilegiado de intercambio entre generaciones». Premios Bancaixa 1993, Valencia: Bancaixa.

BAzo, M.T.; DomíngueZ-AlCón, C. (1996). «Los cuidados familiares de salud en las personas ancianas y las políticas sociales». Revista Española de Investigaciones Sociológicas, 73, p. 43-56.

Fernández Cordón, J.A.; Tobio, C. (1999). «Lone Parent Families in Spain». REIS, edición inglesa, p. 143-179.

Herlyn, I.; Kistner, A. (1997). "Großmuttersein Heute». En Zeitschrift für Frauenforschung, 15. Bielefeld, p. 5-21.

Herlyn, I.; Kistner, A.; LANGer-Schulz, H.; LehmanN, B.; WÄChter, J. (1998). Großmutterschaft im weiblichen Lebenszusammenhang. Eine Untersuchung zu familialen Generationenbeziehungen aus der Perspektive von Großmüttern. Paffenweiler: Centaurus Verlag.

JAMIESON, A.; ILLSLEY, R. (1993). Comparación de politicas europeas de atención a las personas ancianas. Barcelona: S.G. Editores.

JOHnSON, J.; Slater, R. (1993). Aging and later age. Londres: Sage.

KÖNIG, R. (ed.) (1976). Handbuch der emprischen Sozialforschung: Familie, Alter. Band 7. Stuttgart: Enke.

Kunkel, S.; Morgan, L.A. (1998). «Aging. The Social Context». Thousand Oaks, California: Pine Forge Press.

Lamo de Espinosa, E.; Giner, S.; Torres, C. (eds.) (1998). Diccionario de sociología. Madrid: Alianza.

LANGlois, S.; DEL CAMPO, S. (eds.). ¿Convergencia o divergencia? Bilbao: Fundación BBV.

MinisteRIO DE AsunTOS SOCIALES. INSERSO (1995). Las personas mayores en España. Perfiles. Reciprocidad familiar. Madrid.

PFEIFER, S.P.; SUSSMAN, N.B. (eds.) (1994). Families, intergenerational and generational connections. Nueva York: University Press.

RADL Philipp, R. (1988). «La familia como instancia socializadora». Revista Internacional de Sociología, 2.

- (1997). «Familia, educación en contenidos transversales y desarrollo de una identidad reflexiva». En Rosales, C. Innovación Educativa. Santiago de Compostela: Ediciones Tórculo. 
Radl Philipp, R.; García Negro, M. ${ }^{a}$ C. (1995). As mulleres e os cambios sociais e económicos. Santiago de Compostela: Universidade de Santiago.

RichTER, U. (1994). Was heißt hier Oma. Das Selbstverständnis der Großmütter heute. Stuttgart: Klett.

SÁnchez Vera, P. (1993). Sociedad y población anciana. Murcia: Serv. Public. Universidad de Murcia.

SANTOS DEL CAMPO, I. (1996). «Envejecimiento demográfico: diferencias por género». Revista Española de Investigaciones Sociológicas, 73, enero-marzo, p. 177-190.

SOMmer-Himmel, R. (2000). Grosseltern heute. Betreuen, erziehen, verwöhnen. Eine qualitative Studie zum Betreunngsalltag mit Enkelkindern. Kleine Verlag, Bielefeld.

VALERO, A. (coord.) (1995). «Monográfico sobre la familia. Nuevas estrategias y nuevas solidaridades». Revista Española de Investigaciones Sociológicas, 70, abril-junio.

WALKER, A. (1996). «Actitudes europeas ante el envejecimiento y las personas mayores». Revista Española de Investigaciones Sociológicas, 73, enero-marzo, p. 17-42. 